\title{
Spanning trails in a 2-connected graph
}

\author{
Shipeng Wang Liming Xiong \\ School of Mathematics and Statistics \\ Beijing Key Laboratory on MCAACI \\ Beijing Institute of Technology \\ Beijing, 100081, P.R. China \\ spwang22@yahoo.com, lmxiong@bit.edu.cn
}

Submitted: Aug 24, 2018; Accepted: Jul 3, 2019; Published: Sep 27, 2019

(C) The authors. Released under the CC BY-ND license (International 4.0).

\begin{abstract}
In this article we prove the following: Let $G$ be a 2-connected graph with circumference $c(G)$. If $c(G) \leqslant 5$, then $G$ has a spanning trail starting from any vertex, if $c(G) \leqslant 7$, then $G$ has a spanning trail.

As applications of this result, we obtain the following.

(1) Every 2-edge-connected graph of order at most 8 has a spanning trail starting from any vertex with the exception of six graphs.

(2) Let $G$ be a 2-edge-connected graph and $S$ a subset of $V(G)$ such that $E(G-$ $S)=\emptyset$ and $|S| \leqslant 6$. Then $G$ has a trail traversing all vertices of $S$ with the exception of two graphs, moreover, if $|S| \leqslant 4$, then $G$ has a trail starting from any vertex of $S$ and containing $S$.

(3) Every 2-connected claw-free graph $G$ with order $n$ and minimum degree $\delta(G)>$ $\frac{n}{7}+4 \geqslant 23$ is traceable or belongs to two exceptional families of well-defined graphs, and moreover, if $\delta(G)>\frac{n}{6}+4 \geqslant 13$, then $G$ is traceable.
\end{abstract}

All above results are sharp in a sense.

Mathematics Subject Classifications: 05C38, 05C45

\section{Introduction}

A graph $G$ is simple if it has no loops or parallel edges, otherwise we say that $G$ is a multigraph. We consider finite simple undirected graphs $G=(V(G), E(G))$, and for concepts and notations not defined here we refer to [1].

Let $G$ be a graph, and let $H_{1}, H_{2}$ be two subgraphs of $G$. For a vertex $v \in V(G)$, we define $N_{H_{1}}(v)=\left\{u \in V\left(H_{1}\right) \mid u v \in E(G)\right\}$. We define $N_{H_{2}}\left(H_{1}\right)=\bigcup_{v \in V\left(H_{1}\right)} N_{H_{2}}(v)$. The degree $v$ in $H_{1}$ is denoted $d_{H_{1}}(v)=\left|N_{H_{1}}(v)\right|$. The circumference of $G$, denoted by $c(G)$, is 
the length of a longest cycle of $G$. For $S \subset V(G)$ (or $S \subset E(G)$ ), we use $\langle S\rangle_{G}$ to denote the subgraph of $G$ induced by $S$. A pendant vertex of a graph is a vertex of degree 1 , and a pendant edge is an edge having a pendant vertex as an end vertex.

Let $G$ be a graph and $H$ a subgraph of $G$. For $u, v \in V(H)$, the distance between $u$ and $v$ in $H$, denoted $\operatorname{dist}_{H}(u, v)$, the length of a shortest path between $u$ and $v$ in $H$. The contraction $G / H$ is the graph obtained from $G$ by replacing $H$ by a vertex $v_{H}$ such that the number of edges in $G / H$ joining any $v \in V(G-V(H))$ to $v_{H}$ in $G / H$ equal to the number of edges joining $v$ in $G$ to $H$. A graph $G$ is contractible to a graph $G^{\prime}$ if $G$ contains pairwise vertex-disjoint connected subgraphs $H_{1}, \cdots, H_{t}$ with $\bigcup_{i=1}^{t} V\left(H_{i}\right)=V(G)$ such that $G^{\prime}$ is obtained from $G$ by successive contracting each $H_{i}(1 \leqslant i \leqslant t)$. Each subgraph $H \in\left\{H_{1}, \cdots, H_{t}\right\}$ is called the preimage of the vertex $v_{H}$ of $G^{\prime}$. A vertex $v_{H}$ in $G^{\prime}$ is nontrivial if $v_{H}$ is the contraction image of a nontrivial connected subgraph $H$ of $G$.

A graph is called hamiltonian if it contains a Hamilton cycle, i.e., a cycle containing all its vertices. A graph is called traceable if it contains a Hamilton path, i.e., a path containing all its vertices. A trail in a graph $G$ is a sequence $W:=v_{0} e_{1} v_{2} \cdots v_{l-1} e_{l} v_{l}$, whose terms are alternately vertices (not necessarily distinct) and distinct edges of $G$, such that $v_{i-1}$ and $v_{i}$ are ends of $e_{i}$ for $1 \leqslant i \leqslant l$. For convenience, we sometimes abbreviate the term $v_{0} e_{1} v_{1} \cdots v_{l-1} e_{l} v_{l}$ to $v_{0} v_{1} \cdots v_{l-1} v_{l}$. A spanning trail of a graph $G$ is a trail that contains all vertices of $G$. For a subset $S \subseteq V(G)$, if a trail of $G$ traverses all vertices of $S$, then we call it $S$-trail. A subgraph $H$ of a graph $G$ is dominating if every edge of $G$ has at least one end in $H$. A a graph $G$ is even if every vertex of $G$ has even degree. If $H$ is a graph, then the line graph of $H$, denoted $L(H)$, is the graph with $E(H)$ as vertex set, in which two vertices are adjacent if and only if the corresponding edges have a vertex in common.

A vertex $x \in V(G)$ is locally connected if the neighborhood of $x$ induces a connected subgraph in $G$. For $x \in V(G)$, the graph $G_{x}^{\prime}$ obtained from $G$ by adding the edges $\left\{y z: y, z \in N_{G}(x)\right.$ and $\left.y z \notin E(G)\right\}$ is called the local completion of $G$ at $x$. The closure of a claw-free graph $G$, denoted by $\mathrm{cl}(\mathrm{G})$, is obtained from $G$ by recursively performing local completions at any locally connected vertex with non-complete neighborhood, as long as it is possible. If $G$ is a claw-free graph such that $G=\operatorname{cl}(\mathrm{G})$, then we say that $G$ is closed.

A well-known result on spanning closed trail was obtained by Jaeger, later proved independently by Catlin.

Theorem 1. (Catlin [4] and Jaeger [12]) Every 4-edge-connected graph has a spanning closed trail.

Lai et al. presented a sufficient condition for spanning closed trail of 3-edge-connected graphs involved circumference, which was used in hamiltonian claw-free graphs in the same paper.

Theorem 2. (Lai et al. [16]) Every 3-edge-connected graph $G$ with $c(G) \leqslant 8$ has a spanning closed trail.

The above result is sharp because the Pertersen graph has circumference 9 without any spanning closed trail. Chen et al. studied the existence of a spanning closed trail $H$ 


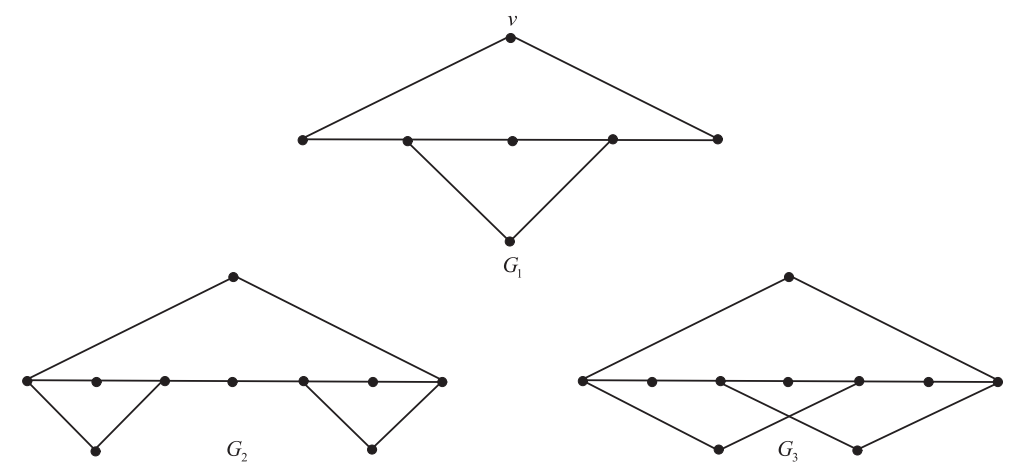

Figure 1: $G_{1}$ has no spanning trail starting from $v, G_{2}$ and $G_{3}$ have no spanning trail.

of 3-edge-connected graphs $G$ such that $H$ contains a given set of vertices of $G$, which was also used in the hamiltonian line graphs in their paper.

Theorem 3. (Chen et al. [6]) Let $G$ be a 3-edge-connected graph and let $S \subseteq V$ be a vertex subset such that $|S| \leqslant 12$. Then either $G$ has a spanning closed trail $H$ such that $S \subseteq V(H)$, or $G$ can be contracted to the Petersen graph in such a way that the preimage of each vertex of the Petersen graph contains at least one vertex in $S$.

Finding similar sufficient conditions for the existence of a spanning closed trail in a 2-edge-connected graph is somewhat trivial because $K_{2,3}$ is a counter-example even for the case $|S|=3$. However, it is nontrivial to use the circumference condition to study the existence of a spanning trail of 2-connected graphs, we prove the following in this paper.

Theorem 4. Let $G$ be a 2-connected graph. Then

(1) if $c(G) \leqslant 5$, then $G$ has a spanning trail starting from any vertex,

(2) if $c(G) \leqslant 7$, then $G$ has a spanning trail.

Theorem 5. Let $G$ be a 2-edge-connected graph and $S$ a subset of $V(G)$ such that $E(G-$ $S)=\emptyset$ and $|S| \leqslant 6$. Then $G$ has an $S$-trail or $G \in\left\{G_{2}, G_{3}\right\}$, where $G_{2}, G_{3}$ are shown in Figure 1, moreover, if $|S| \leqslant 4$, then $G$ has an $S$-trail starting from any vertex of $S$.

Theorem 4 is sharp because of graphs $G_{1}, G_{2}, G_{3}$ shown in Figure 1. For more results involved spanning trail we refer to [3][5][8][15][18]. In [22], Tian et al. applied Theorem 4 to prove that every 2-edge-connected graph of order at most 11 has a spanning trail with the exception of six graphs. We also apply Theorem 4 to prove the similar result on special spanning trail.

Theorem 6. Let $G$ be a 2-edge-connected graph. If $|V(G)| \leqslant 8$, then either $G$ has a spanning trail starting from any vertex or $G \in\left\{F_{1}, F_{2}, F_{3}, F_{4}, F_{5}, F_{6}\right\}$, where $F_{i}$ is shown in Figure 2. Moreover, if $G \in\left\{F_{1}, F_{2}, F_{3}, F_{4}, F_{5}\right\}$, then $G$ has no spanning trail starting from any vertex in $\left\{u, x_{1}, x_{2}\right\}$, but has a spanning trail starting from any vertex in $\left\{u_{1}, u_{2}, \cdots, u_{7}\right\}$. 

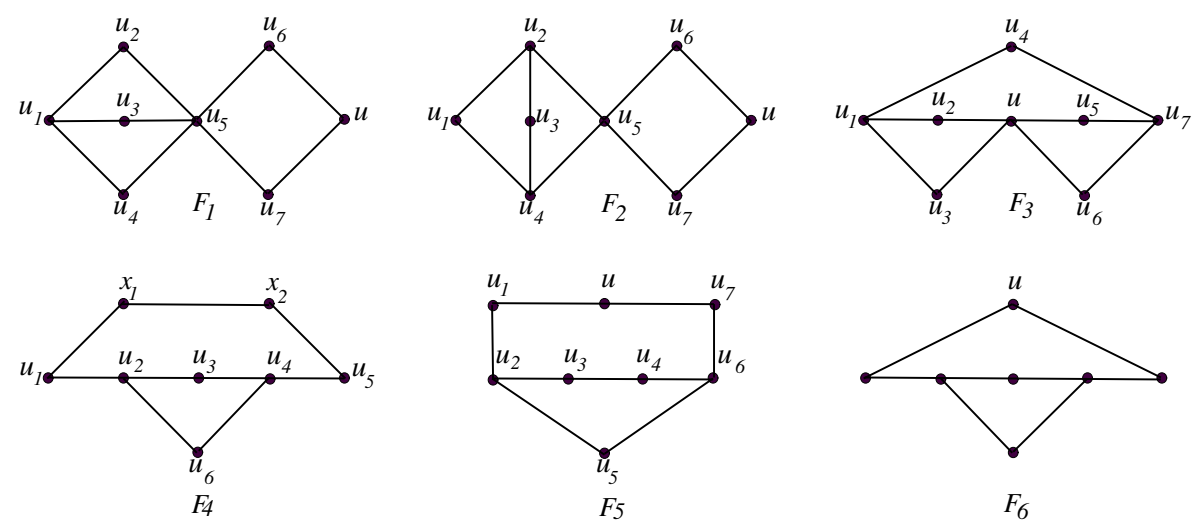

Figure $2: F_{i}(1 \leqslant i \leqslant 6)$ is a graph of order at most 8 that has no spanning trail starting from $x_{1}, x_{2}$ and $u$, but has a spanning trail starting from any vertex in $V\left(F_{i}\right) \backslash\left\{x_{1}, x_{2}\right\}$.

Actually, we use Theorems 4 and 6 to prove Theorem 5. As an application of Theorem 5 , we obtain the following result (i.e., Theorem 7). We believe that Theorem 4 may have further applications in similar researches, as shown in the concluding remarks.

Before stating the following result, we need to define two families of graphs.

$\mathcal{C}_{1}=\left\{H: H\right.$ is obtained from $G_{2}$ shown in Figure 1, by adding at least one pendant edge to each vertex of degree two $\}$,

$\mathcal{C}_{2}=\left\{H: H\right.$ is obtained from $G_{3}$ shown in Figure 1, by adding at least one pendant edge to each vertex of degree two $\}$.

Theorem 7. Let $G$ be a 2-connected claw-free graph of order $n \geqslant 133$ such that $\delta(G) \geqslant$ $\frac{n}{7}+4$. Then $G$ is traceable or $\operatorname{cl}(\mathrm{G})=L(H)$ where $H \in \mathcal{C}_{1} \cup \mathcal{C}_{2}$.

As a corollary of Theorem 7, we prove the following result.

Corollary 8. Let $G$ be a 2 -connected claw-free graph of order $n \geqslant 54$ with $\delta(G)>\frac{n}{6}+4$. Then $G$ is traceable.

In fact, the results on claw-free graph are already known in [14], it is enough to check the exceptions for traceability. But only computer proofs were known so far.

In the next section, we will present some necessary results involved Ryjáček closure and Catlin reduction. In Section 3, we will complete the proof of Theorem 4. In Sections 4 and 5, we will prove Theorems 6 and 5, respectively. In Section 6, we will prove Theorem 7 and Corollary 8. In the final section, we will give some concluding remarks.

\section{Preliminaries and basic results}

\subsection{Ryjáček closure}

Ryjáček [20] first investigated the closure of a claw-free graph $G$, which becomes a useful tool in investigating hamiltonian properties of claw-free graphs. And in [20], he proved the following well-known theorem. 
Theorem 9. (Ryjáček [20]) Let $G$ be a claw-free graph. Then

(1) $\operatorname{cl}(\mathrm{G})$ is uniquely determined,

(2) $\operatorname{cl}(\mathrm{G})$ is claw-free,

(3) $\mathrm{cl}(\mathrm{G})$ is the line graph of a triangle-free graph.

Theorem 10. (Brandt, Favaron and Ryjáček [2]) Let $G$ be a claw-free graph. Then $G$ is traceable if and only if $\mathrm{cl}(\mathrm{G})$ is traceable.

\subsection{Catlin reduction}

For a graph $G$ and a subgraph $H$ of $G$, let $O(H)$ denote the set of all odd degree vertices in $H$. We say that $G$ is collapsible if for every subset $X \subseteq V(G)$ with $|X|$ even, there is a spanning connected subgraph $H_{X}$ of $G$ such that $O\left(H_{X}\right)=X$. In [4], Catlin showed that every graph $G$ has a unique collection of pairwise vertex-disjoint maximal collapsible subgraphs $H_{1}, \cdots, H_{t}$ such that $\bigcup_{i=1}^{t} V\left(H_{i}\right)=V(G)$. The reduction $G^{\prime}$ of a graph $G$ is the graph obtained from $G$ by contracting each maximal collapsible subgraph $H_{i}(1 \leqslant i \leqslant t)$ into a single vertex $v_{i}$.

Theorem 11. (Catlin [4]) Let $G$ be a connected graph. Then the reduction of $G$ is a simple graph and has no cycle of length less than four.

Theorem 12. (Catlin [4]) Let $G$ be a connected graph and $H$ a collapsible subgraph of $G$. Then $G$ has a spanning closed trail if and only if $G / H$ has a spanning closed trail.

Theorem 13. (Xiong, et al. [25]) Let $G$ be a connected graph and $G^{\prime}$ the reduction of $G$. Then $G$ has a spanning trail if and only if $G^{\prime}$ has a spanning trail.

We prove a similar result on special spanning trail.

Theorem 14. Let $G$ be a connected graph and $G^{\prime}$ the reduction of $G$. Then $G$ has a spanning trail starting from any vertex of $G$ if and only if $G^{\prime}$ has a spanning trail starting from any vertex of $G^{\prime}$.

Proof. $G$ has a spanning trail starting from any vertex if and only if for any collapsible subgraph $H$ of $G, G / H$ has a spanning trail starting from any vertex in $G / H$. Note that a graph has a spanning trail $T$ starting from $v$ if and only if one can add at most one edge $e_{v}$ to create a spanning closed trail. Also note that $G+e_{v}$ has a spanning closed trail if and only if $\left(G+e_{v}\right) / H$ has a spanning closed trail for any collapsible subgraph $H$. Thus Theorem 12 implies that Theorem 14 holds. 


\section{The proof of Theorem 4}

Let $G$ be a 2-connected graph and $C$ a cycle of $G$. Then every component $D$ of $G-V(C)$ has at least two distinct neighbors on $C$. For any path $P$ in $D$, if the two ends (possibly only one if $P$ is a vertex) of $P$ have two distinct neighbors $x_{1}, x_{2}$ on $C$, then $P$ is called a 2-attaching path of $C$ in $D$, and these two vertices $x_{1}, x_{2}$ are called a 2-attaching pair of $P$ on $C$. We observe that if $D \cong K_{1}$ or $K_{2}$, then $D$ is the 2-attaching path of $C$. Furthermore, if $D$ has a longest 2-attaching path $P$ of order $k$, then $D$ is called a $k$ component of $G-V(C)$. For any cycle of a graph $G$, we can give $C$ an orientation $\vec{C}$. We can bound any component of $G-V(C)$ by the circumference $c(G)$.

Lemma 15. Let $G$ be a 2-connected graph with circumference $c(G)$ and $C$ a longest cycle of $G$. Then

(1) every $k$-component $D$ of $G-V(C)$ holds $k \leqslant\left\lfloor\frac{c(G)}{2}\right\rfloor-1$,

(2) if $c(G) \leqslant 5$, then every vertex $v$ of $G$ lies on a longest cycle.

Proof. Suppose, by contradiction, that $D$ is a $k$-component of $G-V(C)$ with $k \geqslant\left\lfloor\frac{c(G)}{2}\right\rfloor$. Then $C$ has a 2-attaching path $P_{k}\left(k \geqslant\left\lfloor\frac{c(G)}{2}\right\rfloor\right)$ in $D$ with a 2-attaching pair $x_{1}, x_{2}$, thus either $x_{1} \vec{C} x_{2} P_{k} x_{1}$ or $x_{1} \overleftarrow{C} x_{2} P_{k} x_{1}$ is a cycle of length at least $k+\left\lfloor\frac{c(G)}{2}\right\rfloor+1>c(G)$, a contradiction. This proves Lemma 15(1).

We now show (2), it suffices to consider the case when $v \notin V(C)$. Since $c(G) \leqslant 5$, by Lemma 15(1), $v$ is a 1-component of $G-V(C)$ and let $x_{1}, x_{2}$ be a 2-attaching pair of $v$ on $C$. Then either $x_{1} \vec{C} x_{2} v x_{1}$ or $x_{1} \overleftarrow{C} x_{2} v x_{1}$ is a cycle of length $c(G)$, we are done.

We denote by $G\left[x ; y_{1}, y_{2}, \cdots, y_{t}\right]$ a star with $x$ as its center and $y_{1}, y_{2}, \cdots, y_{t}$ are its leaves.

Lemma 16. Let $G$ be a 2-connected graph and $C$ a longest cycle of $G$, and let $D$ be a 2-component of $G-V(C)$. Then

(1) $D$ is a star, denoted by $G\left[x ; y_{1}, y_{2}, \cdots, y_{t}\right]$,

(2) if $6 \leqslant|V(C)| \leqslant 7$ and $t=1$, then $\left|N_{G}(D) \cap V(C)\right|=2$ and $2 \leqslant d_{G}\left(y_{1}\right) \leqslant 3$,

(3) if $6 \leqslant|V(C)| \leqslant 7$ and $t \geqslant 2$, then $\left|N_{G}(D) \cap V(C)\right|=2$ and $d_{G}\left(y_{i}\right)=2$, for $1 \leqslant i \leqslant t$.

Proof of Lemma 16. Let $C=v_{0} v_{1} \cdots v_{c(G)-1} v_{0}$ be a longest cycle of $G$. We have the following fact.

Claim 17. $D$ is a tree.

Proof. Suppose, by contradiction, that there is a cycle $C^{\prime}$ in $D$. Since $G$ is 2-connected, there exist two pairwise disjoint paths in $G$ joining $C^{\prime}$ and $C$. This yields a 2-attaching path of $C$ in $D$ containing at least three vertices of $C^{\prime}$, which contradicts the fact that $D$ is a 2-component of $G-V(C)$. This proves Claim 17 . 
Claim 18. $\operatorname{diam}(D) \leqslant 2$.

Proof. Suppose to the contrary that $\operatorname{diam}(D) \geqslant 3$. Then there is a diameter path $P=$ $x_{1} \cdots x_{k}$ in $D$ with $k \geqslant 4$. By Claim $17, D$ is a tree and hence $x_{1}, x_{k}$ are two leaves of $D$. Since $G$ is 2-connected, each $x_{i}$ has a neighbor on $C$ for $i=1, k$. Since $D$ is a 2-component of $G-V(C), P$ cannot be a 2-attaching path of $C$ in $D$, implying that $\left|\left(N_{G}\left(x_{1}\right) \cup N_{G}\left(x_{k}\right)\right) \cap V(C)\right|=1$, say $\left(N_{G}\left(x_{1}\right) \cup N_{G}\left(x_{k}\right)\right) \cap V(C)=\{v\}$. Since $G$ is 2-connected, there exist two pairwise disjoint paths $Q_{1}, Q_{2}$ in $G$ joining $P$ and $C$, then one of them joins some internal vertex of $P$ and some vertex of $V(C) \backslash\{v\}$. This yields a 2-attaching path of $D$ containing at least three vertices, which contradicts the fact that $D$ is a 2-component of $G-V(C)$. This proves Claim 18.

By Claims 17 and 18, $D$ is a star. This proves Lemma 16(1). Let $D \cong G\left[x ; y_{1}, y_{2}, \cdots, y_{t}\right]$ be a star. Since $t=1, D$ is an edge $x y_{1}$. It is easy to see that $x y_{1}$ is the 2 -attaching path of $C$. Let $x_{1}, x_{2}$ be a 2-attaching pair of $x y_{1}$ on $C$. Then $\operatorname{dist}_{C}\left(x_{1}, x_{2}\right) \geqslant 3$, otherwise either $x_{1} \vec{C} x_{2} x y_{1} x_{1}$ or $x_{1} \overleftarrow{C} x_{2} x y_{1} x_{1}$ is a cycle of length at least $c(G)+1$. Hence, by $6 \leqslant|V(C)| \leqslant 7$ we have $\operatorname{dist}_{C}\left(x_{1}, x_{2}\right)=3$. Therefore, since $C$ is a longest cycle of $G$, $N_{G}(D) \cap V(C)=\left\{x_{1}, x_{2}\right\}$. This proves Lemma 16(2).

Since $G$ is 2 -connected and $D$ is a star, $N_{G}\left(y_{i}\right) \cap V(C) \neq \emptyset$ for $1 \leqslant i \leqslant t$. By the definition of 2-component, $N_{G}\left(y_{i}\right) \cap V(C)$ and $N_{G}\left(y_{j}\right) \cap V(C)$ have the same vertex $v_{0}$ (say) for any pair of $\{i, j\} \subseteq\{1,2, \cdots, t\}$, this implies that $d_{G}\left(y_{i}\right)=2$ for $1 \leqslant i \leqslant t$. Since $G$ is 2-connected, $\left(N_{G}(x) \cap V(C)\right) \backslash\left\{v_{0}\right\} \neq \emptyset$, then $x y_{i}$ is a 2-attaching path of $C$ in $D$ for $1 \leqslant i \leqslant t$. Therefore, since $C$ is a longest cycle of $G,\left|\left(N_{G}(x) \cap V(C)\right) \backslash\left\{v_{0}\right\}\right|=1$. Then, since $N_{G}\left(y_{i}\right) \cap V(C)=N_{G}\left(y_{j}\right) \cap V(C)=\left\{v_{0}\right\}$ for any pair of $\{i, j\} \subseteq\{1,2, \cdots, t\}$, we have $\left|N_{G}(D) \cap V(C)\right|=2$. This proves Lemma 16(3).

Let $G$ be a 2-connected graph and $C$ a cycle of $G$. Let $D_{1}$ and $D_{2}$ be two components of $G-V(C)$ and let $P, P^{\prime}$ be two 2-attaching paths of $C$ in $D_{1}$ and $D_{2}$ with two 2-attaching pairs $\left\{x_{1}, x_{2}\right\}$ and $\left\{x_{3}, x_{4}\right\}$, respectively. If $x_{1}, x_{3}, x_{2}, x_{4}$ are four distinct vertices that lie along the direction of $\vec{C}$, then we say that $D_{1}$ overlaps $D_{2}$ on $C$. If $\left\{x_{1}, x_{2}\right\}=\left\{x_{3}, x_{4}\right\}$, then we say that $D_{1}$ is equivalent to $D_{2}$.

Proof of Theorem 4. Let $C=v_{0} v_{1} v_{2} \cdots v_{c(G)-1} v_{0}$ be a longest cycle of $G$. By deleting all chords of $C$, the resulting 2-connected graph $G_{1}$ is a spanning subgraph of $G$. It suffices to show that $G_{1}$ has a spanning trail. If $V\left(G_{1}\right) \backslash V(C)=\emptyset$, then $C$ is our desired spanning connected even subgraph. Hence we assume that $V\left(G_{1}\right) \backslash V(C) \neq \emptyset$ and we have the following fact.

Claim 19. Any pair of components of $G_{1}-V(C)$ cannot overlap on $C$.

Proof. Suppose, by contradiction, that there is a pair of components $D_{1}, D_{2}$ in $G_{1}-V(C)$ such that they overlap each other. Then there exist two 2-attaching paths $P$ and $P^{\prime}$ of $C$ in $D_{1}$ and $D_{2}$ with two 2-attaching pairs $\left\{x_{1}, x_{2}\right\},\left\{x_{3}, x_{4}\right\}$ on $C$, respectively, such that $x_{1}, x_{3}, x_{2}, x_{4}$ are four distinct vertices along the orientation $\vec{C}$. Therefore, either 
$x_{1} P x_{2} \overleftarrow{C} x_{3} P^{\prime} x_{4} \vec{C} x_{1}$ or $x_{1} P x_{2} \vec{C} x_{4} P^{\prime} x_{3} \overleftarrow{C} x_{1}$ is a cycle of length at least $\left\lceil\frac{|V(C)|}{2}\right\rceil+4$, then $\left\lceil\frac{|V(C)|}{2}\right\rceil+4>|V(C)|$ for $|V(C)| \leqslant 7$, a contradiction. This proves Claim 19.

Since $C$ is a longest cycle of $G$, we clearly have the following two facts.

Claim 20. For any 2-attaching path $P$ of $C$ and for each 2-attaching pair $x_{1}, x_{2}$ of $P$, it holds $\operatorname{dist}_{C}\left(x_{1}, x_{2}\right) \geqslant 2$.

Claim 21. For any 2-component $D$ of $G_{1}-V(C)$ and for any longest 2-attaching path $P$ of $C$ in $D$, it holds $\operatorname{dist}_{C}\left(x_{1}, x_{2}\right) \geqslant 3$ for each 2-attaching pair $x_{1}, x_{2}$ of $P$.

We first show Theorem 4(1). Fix a vertex $v$ in $G$, it suffices to show that $G_{1}$ has a spanning trail starting from $v$. By Lemma 15(2), $v$ lies on a longest cycle of $G$. Without loss of generality, we may assume that $v \in V(C)$. Let $X$ be the set of all vertices in $G_{1}$ with degree odd. Since $c\left(G_{1}\right) \leqslant 5$ and $C$ is a longest cycle of $G_{1}$, every component of $G_{1}-V(C)$ is a 1-component by Lemma 15(1). Again, since $C$ is a longest cycle of $G$, every component of $G_{1}-V(C)$ has exactly two neighbors on $C$, implying that $X \subseteq V(C)$. If $|X|=4$, then there exist four vertices of $V(C)$ that are consecutive on $C$. Without loss of generality, we may assume that say $X=\left\{v_{0}, v_{1}, v_{2}, v_{3}\right\}$. Since $C$ has no chord and every component of $G_{1}-V(C)$ has exactly two neighbors on $C$ and by Claim 20, there exist two components $w_{1}, w_{2}$ of $G_{1}-V(C)$ such that $N_{G}\left(w_{1}\right) \cap\left\{v_{0}, v_{1}, v_{2}, v_{3}\right\}=\left\{v_{0}, v_{2}\right\}$ and $N_{G}\left(w_{2}\right) \cap\left\{v_{0}, v_{1}, v_{2}, v_{3}\right\}=\left\{v_{1}, v_{3}\right\}$, then $w_{1}$ overlaps $w_{2}$, contradicting Claim 19. Hence we have $|X|<4$, then $|X|=0,2$, it suffices to consider the case when $|X|=2$ (if $|X|=0$, then $G_{1}$ is a connected even graph, clearly $G_{1}$ has a spanning trail starting from $v$ ). If $v \in X$, then $G_{1}$ obviously has a spanning trail starting from $v$.

Hence we assume that $v \notin X$ and let $X=\left\{v_{i}, v_{j}\right\}$ with $i \neq j$. If $v_{i} v_{j} \in E(C)$, then $G_{1} \backslash v_{i} v_{j}$ is a connected even graph, clearly $G_{1}$ has a spanning trail starting from $v$. Hence we assume that $v_{i} v_{j} \notin E(C)$, then by $|V(C)| \leqslant 5$, there is a vertex in $\left\{v_{i}, v_{j}\right\}$, say $v_{i}$, that is adjacent to $v$, then $G_{1} \backslash v v_{i}$ is a connected spanning subgraph of $G_{1}$ with exactly two odd degree vertices $v, v_{i}$, and hence $G$ has a spanning trail starting from $v$. This proves Theorem 4(1).

We now show Theorem 4(2). By Theorem 4(1), it suffices to consider the case $6 \leqslant$ $c(G) \leqslant 7$. Since $C$ is a longest cycle of $G_{1}$ and by Lemma 16(2)-(3), we have the following.

Claim 22. Every 2-component of $G_{1}-V(C)$ has exactly two neighbors on $C$.

Claim 23. For any pair of 2-components $D_{1}$ and $D_{2}$ of $G_{1}-V(C)$, it holds that $N_{G_{1}}\left(D_{1}\right) \cap$ $N_{G_{1}}\left(D_{2}\right) \cap V(C) \neq \emptyset$.

Proof. Suppose, by contradiction, that there is a pair of 2-components $D_{1}, D_{2}$ of $G_{1}-V(C)$ such that $N_{G_{1}}\left(D_{1}\right) \cap N_{G_{1}}\left(D_{2}\right) \cap V(C)=\emptyset$. Then by Claim 22, there exist four distinct vertices $v_{i}, v_{j}, v_{k}, v_{l}$ on $C$ such that $v_{i}, v_{j}$ and $v_{k}, v_{l}$ are two neighbors of $D_{1}$ and $D_{2}$, respectively. By Claim 19, we may, without loss of generality, assume that $0 \leqslant i<j<$ $k<l \leqslant c(G)-1$. Since $C$ is a longest cycle of $G$ and by the definition of 2-component, $\operatorname{dist}_{C}\left(v_{i}, v_{j}\right) \geqslant 3$ and $\operatorname{dist}_{C}\left(v_{k}, v_{l}\right) \geqslant 3$. This implies that $j-i \geqslant 3$ and $l-k \geqslant 3$, hence $|V(C)| \geqslant 8$, a contradiction. This proves Claim 23 . 
Claim 24. The neighbors of all 2-components of $G_{1}-V(C)$ on $C$ are at most three.

Proof. If all 2-components of $G_{1}-V(C)$ are equivalent each other, then by Claim 22, the neighbors of all 2-components of $G_{1}-V(C)$ on $C$ are exactly two, we are done.

Hence we assume that there is a pair of 2-components $D_{1}$ and $D_{2}$ of $G_{1}-V(C)$ such that they are not equivalent. By Claims 22 and 23, $\left|N_{G_{1}}\left(D_{1}\right) \cap N_{G_{1}}\left(D_{2}\right) \cap V(C)\right|=1$, say $N_{G_{1}}\left(D_{1}\right) \cap V(C)=\left\{v_{0}, v_{i}\right\}$ and $N_{G_{2}}\left(D_{1}\right) \cap V(C)=\left\{v_{0}, v_{j}\right\}$ with $0<i<j$. By Claim 21, we have $\operatorname{dist}_{C}\left(v_{0}, v_{i}\right) \geqslant 3$ and $\operatorname{dist}_{C}\left(v_{0}, v_{j}\right) \geqslant 3$. Hence, by $6 \leqslant c(G) \leqslant 7$ and $i<j$, we have $c(G)=7$ and then $i=3, j=4$.

We will show that the neighbors of all 2-components of $G_{1}-V(C)$ on $C$ belong to $\left\{v_{0}, v_{3}, v_{4}\right\}$ to complete the remaining part of the claim, it is suffices to show that any 2-component of $G_{1}-V(C)$ is equivalent to $D_{1}$ or $D_{2}$. Suppose not. Then $G_{1}-V(C)$ has a 2-component $D_{3}$ is neither equivalent to $D_{1}$ nor to $D_{2}$, thus $\left(N_{G}\left(D_{3}\right) \cap V(C)\right) \backslash\left\{v_{0}, v_{3}, v_{4}\right\} \neq$ $\emptyset$, say $v_{k} \in\left(N_{G}\left(D_{3}\right) \cap V(C)\right) \backslash\left\{v_{0}, v_{3}, v_{4}\right\}$. This implies that $v_{k} \in\left\{v_{1}, v_{2}\right\}$ or $v_{k} \in\left\{v_{5}, v_{6}\right\}$. Up to symmetry, we may assume that $v_{k} \in\left\{v_{1}, v_{2}\right\}$, then by Claims 19 and 22, there is only one vertex $v_{l} \in\left(N_{G_{1}}\left(D_{3}\right) \cap V(C)\right) \backslash\left\{v_{k}\right\}$ such that $v_{l} \notin\left\{v_{4}, v_{5}, v_{6}\right\}$ and hence $v_{l} \in$ $\left\{v_{0}, v_{1}, v_{2}, v_{3}\right\} \backslash\left\{v_{k}\right\}$. Since $v_{k}, v_{l} \in N_{G_{1}}\left(D_{3}\right) \cap V(C)$, by Claim 22, there is a 2-attaching path $P$ of $C$ in $D_{3}$ with 2-attaching pair $v_{k}, v_{l}$, then either $v_{k} P v_{l} \vec{C} v_{k}$ or $v_{k} P v_{l} \overleftarrow{C} v_{k}$ is a cycle of length at least 8, a contradiction. This proves Claim 24.

By Lemma 16(1), every 2-component of $G_{1}-V(C)$ is a star. By Claim 24, we may assume that $v_{i^{\prime}}, v_{i^{\prime \prime}}, v_{i^{\prime \prime \prime}}$ are neighbors of all 2-components of $G_{1}-V(C)$ on $C$ and $2 \leqslant\left|\left\{v_{i^{\prime}}, v_{i^{\prime \prime}}, v_{i^{\prime \prime \prime}}\right\}\right| \leqslant 3$. By Claim 22 , every 2-component of $G_{1}-V(C)$ has exactly two neighbors on $C$. By Claim 21 and $|V(C)| \leqslant 7$, no triple of 2-components $F_{1}, F_{2}, F_{3}$ of $G_{1}-V(C)$ holds that $N_{G_{1}}\left(F_{1}\right) \cap V(C)=\left\{v_{i^{\prime}}, v_{i^{\prime \prime}}\right\}$ and $N_{G_{1}}\left(F_{2}\right) \cap V(C)=$ $\left\{v_{i^{\prime \prime}}, v_{i^{\prime \prime \prime}}\right\}$ and $N_{G_{1}}\left(F_{3}\right) \cap V(C)=\left\{v_{i^{\prime}}, v_{i^{\prime \prime \prime}}\right\}$. Without loss of generality, we may let $\mathcal{H}_{1}=\left\{D_{1}, D_{2}, \cdots, D_{s_{1}}\right\}$ be the set of all 2-components of $G_{1}-V(C)$ such that $N_{G_{1}}\left(D_{j}\right) \cap$ $V(C)=\left\{v_{i^{\prime}}, v_{i^{\prime \prime}}\right\}$ and let $\mathcal{H}_{2}=\left\{D_{s_{1}+1}, D_{s_{1}+2}, \cdots, D_{s_{2}}\right\}$ be the set of all 2-components of $G_{1}-V(C)$ such that $N_{G_{1}}\left(D_{j}\right) \cap V(C)=\left\{v_{i^{\prime \prime}}, v_{i^{\prime \prime \prime}}\right\}$, where $D_{j} \cong G\left[x_{j} ; y_{j, 1}, y_{j, 2}, \cdots, y_{j, t_{j}}\right]$. Let $\mathcal{H}_{3}=\left\{D_{s_{2}+1}, D_{s_{2}+2}, \cdots, D_{s_{3}}\right\}$ be the set of all 1-components of $G_{1}-V(C)$. Since $6 \leqslant c\left(G_{1}\right) \leqslant 7$ and $C$ is a longest cycle of $G_{1}, G_{1}-V(C)$ has no $i$-component with $i \geqslant 3$ by Lemma 15(1). This implies that $\mathcal{H}_{1} \cup \mathcal{H}_{2} \cup \mathcal{H}_{3}$ is the set of all components of $G_{1}-V(C)$.

Without lose of generality, let $\mathcal{H}=\left\{D_{1}, \cdots, D_{s}\right\}$ be the set of all 2-components of $G_{1}-V(C)$ such that $D_{j}$ is an edge $x_{j} y_{j, 1}$. Clearly $\mathcal{H} \subseteq \mathcal{H}_{1} \cup \mathcal{H}_{2}$. By Lemma 16(2), $2 \leqslant d_{G_{1}}\left(y_{j, 1}\right) \leqslant 3$ for all $j \in\{1,2, \cdots, s\}$. Let $G_{2}$ be a spanning subgraph of $G_{1}$ obtained from $G_{1}$ by deleting all edges $y_{j, 1} z$ such that $d_{G_{1}}\left(y_{j, 1}\right)=3$ for all $j \in\{1, \cdots, s\}$, where $z \in\left\{v_{i^{\prime}}, v_{i^{\prime \prime}}, v_{i^{\prime \prime \prime}}\right\}$. Then $d_{G_{2}}\left(y_{j, 1}\right)=2$ for all $j \in\{1,2, \cdots, s\}$. For $s+1 \leqslant i \leqslant s_{2}, j \in$ $\left\{1,2, \cdots, t_{i}\right\}$, we have $d_{G_{2}}\left(y_{i, j}\right)=2$ by Lemma 16(3). Again, by Lemma 16(2)-(3), each $x_{j}$ in $D_{j}$ such that $2 \leqslant d_{G_{2}}\left(x_{j}\right) \leqslant 3$ with $1 \leqslant j \leqslant s_{2}$. Let $G_{3}$ be the resulting graph obtained from $G_{2}$ by deleting exactly one edge $x_{j} z$ such that $d_{G_{2}}\left(x_{j}\right)=3$ where $z$ is a neighbor of $x_{j}$ in $\left\{v_{i^{\prime}}, v_{i^{\prime \prime}}, v_{i^{\prime \prime \prime}}\right\}$ for $1 \leqslant j \leqslant s_{2}$. Clearly, $G_{3}$ is a spanning subgraph of $G_{1}$ and $d_{G_{3}}(w)$ is even for any $w \in V\left(\bigcup_{i=1}^{s_{2}} D_{i}\right)$. It suffices to show that $G_{3}$ has a spanning trail. 
For any 1-component $x$ of $G_{3}-V(C)$, it holds that $\left|N_{G_{3}}(x) \cap V(C)\right| \geqslant 2$, and we take exactly two edges $e_{x}, e_{x}^{\prime}$ incident with $x$. Let $E_{1}=\left\{e_{x}, e_{x}^{\prime}: x\right.$ is a 1-component of $\left.G_{3}-V(C)\right\}$, and let $G_{4} \cong\left\langle E\left(G_{3}-\bigcup_{i=s_{2}+1}^{s_{3}} V\left(D_{i}\right)\right) \cup E_{1}\right\rangle_{G_{3}}$. Clearly, $G_{4}$ is a spanning subgraph of $G_{3}$, it suffices to show that $G_{4}$ has a spanning trail. Let $Y$ be the set of all odd degree vertices in $G_{4}$. Then $Y \subseteq V(C)$. Since $|Y| \leqslant 7$, we have $|Y| \in\{0,2,4,6\}$, it suffices to consider the case when $|Y|=4$ or 6 . (if $|Y|=0$ or 2 , then $G_{4}$ obviously has a spanning trail). Suppose first that $|Y|=6$. Without loss of generality, we may assume that $Y=\left\{v_{0}, v_{1}, v_{2}, v_{3}, v_{4}, v_{5}\right\}$, then $G_{4} \backslash\left\{v_{0} v_{1}, v_{3} v_{4}\right\}$ is a connected spanning subgraph of $G_{4}$ with exactly two odd degree vertices; otherwise $G_{4} \backslash\left\{v_{0} v_{1}, v_{3} v_{4}\right\}$ has two components such that one of them has only one odd degree vertex $v_{2}$, this contradicts the fact that every graph has even number of vertices with degree odd. Therefore, $G_{4} \backslash\left\{v_{0} v_{1}, v_{3} v_{4}\right\}$ has a spanning trail, implying that $G_{4}$ has a spanning trail.

Now suppose that $|Y|=4$. Then, by $|V(C)| \leqslant 7$, there is a pair of vertices $v_{i}, v_{j}$ in $Y$ such that $v_{i} v_{j} \in E(C)$. Therefore, $G_{4} \backslash v_{i} v_{j}$ is a connected spanning subgraph of $G_{4}$ with exactly two odd degree vertices, then $G_{4} \backslash v_{i} v_{j}$ has a spanning trail, therefore, $G_{4}$ has a spanning trail. This completes the proof.

\section{The proof of Theorem 6}

Suppose that there is a vertex $u$ in $G$ that has no spanning trail starting from $u$. It suffices to show that $G \in\left\{F_{1}, F_{2}, F_{3}, F_{4}, F_{5}, F_{6}\right\}$, where $F_{i}$ is shown in Figure 2. Let $G^{\prime}$ be the reduction of $G$. By Theorem 14, $G^{\prime}$ has no spanning trail starting from $u$. By Theorem 11, $G^{\prime}$ is triangle-free.

Suppose first that $\kappa\left(G^{\prime}\right)=1$. Since $G^{\prime}$ is triangle-free, every block of $G^{\prime}$ has at least four vertices. Since $\left|V\left(G^{\prime}\right)\right| \leqslant 8, G^{\prime}$ has exactly two blocks $H_{1}, H_{2}$ with $4 \leqslant\left|V\left(H_{i}\right)\right| \leqslant 5$. If $\left|V\left(H_{1}\right)\right|=\left|V\left(H_{2}\right)\right|=4$, then clearly $G^{\prime} \cong C_{4} \cup C_{4}$ is even, contradicting our hypothesis. Hence one of $H_{1}, H_{2}$ has exactly five vertices. Without loss of generality, we may assume that $\left|V\left(H_{1}\right)\right|=5$. Since $\left|V\left(G^{\prime}\right)\right| \leqslant 8$, we have $\left|V\left(H_{2}\right)\right|=4$, then $H_{2} \cong C_{4}$. Recall that $G^{\prime}$ has no spanning trail starting from $u$, so $H_{1} \nsucceq C_{5}$, otherwise $G^{\prime} \cong C_{5} \cup C_{4}$ is even. Therefore, since $G^{\prime}$ is triangle-free, $H_{1} \cong K_{2,3}$ and then $G^{\prime} \in\left\{F_{1}, F_{2}\right\}$, where $F_{1}, F_{2}$ are shown in Figure 2.

Let us in the following consider the case when $\kappa\left(G^{\prime}\right) \geqslant 2$. Let $C=v_{0} v_{1} \cdots v_{c(G)-1} v_{0}$ be a longest cycle of $G^{\prime}$. Then $c\left(G^{\prime}\right) \leqslant 7$, otherwise $C$ is a Hamilton cycle of $G^{\prime}$. Since $G^{\prime}$ has no spanning trail starting from $u$, by Theorem $4(1)$ we have $c\left(G^{\prime}\right) \geqslant 6$. Since $\left|V\left(G^{\prime}\right)\right| \leqslant 8$ and $6 \leqslant|V(C)| \leqslant 7, G^{\prime}-V(C)$ has at most two vertices. Possibly $G^{\prime}-V(C) \cong K_{1}, K_{2}$ or $2 K_{1}$. Let $\mathcal{H}$ be the set of all components of $G^{\prime}-V(C)$. Then clearly $|\mathcal{H}| \leqslant 2$. For any $F \in \mathcal{H}$, there is a path $P_{F}$ in $G^{\prime}$ joining two distinct vertices of $C$ and containing $V(F)$. Let $G_{1}=\left\langle E(C) \bigcup_{F \in \mathcal{H}} E\left(P_{F}\right)\right\rangle_{G}$. Then $G_{1}$ is a 2-connected spanning subgraph of $G^{\prime}$. Let $X$ be the set of all vertices in $G_{1}$ with degree odd. Then $X \subset V(C)$ and $|X|=2,4$. (If $|X|=0$, then $G_{1}$ is even).

We now distinguish the following two cases. 
Case 1. $|V(C)|=6$.

Case 1.1. $G_{1}-V(C) \cong K_{2}$.

Let $V\left(G_{1}\right) \backslash V(C)=\left\{x_{1}, x_{2}\right\}$. By the definition of $G_{1},|X|=2$ and let $N_{G_{1}}\left(x_{1}\right) \cap$ $V(C)=\left\{v_{i}\right\}$ and $N_{G_{1}}\left(x_{2}\right) \cap V(C)=\left\{v_{j}\right\}$. Since $C$ is a longest cycle of $G_{1}$, we have $\operatorname{dist}_{C}\left(v_{i}, v_{j}\right)=3$. Without loss of generality, we may assume that $v_{i}=v_{0}, v_{j}=v_{3}$, then $E\left(G_{1}\right)=\left\{x_{1} v_{0}, x_{2} v_{3}, x_{1} x_{2}\right\} \cup E(C)$, hence $G_{1}$ has a spanning trail starting from any vertex of $\left\{v_{0}, v_{3}\right\}$. Note that for any vertex $v \in V\left(G_{1}\right) \backslash\left\{v_{0}, v_{3}\right\}$, there is an edge $e \in E\left(G_{1}\right)$ incident with $v$ such that $G_{1} \backslash e$ has exactly two vertices with degree odd. Since $G_{1} \backslash e$ is connected, $G_{1}$ has a spanning trail starting from any vertex in $V\left(G_{1}\right) \backslash\left\{v_{0}, v_{3}\right\}$. Therefore, the above facts imply that $G_{1}$ has a spanning trail starting from any vertex, a contradiction.

Case 1.2. $G_{1}-V(C) \cong 2 K_{1}$.

Let $V\left(G_{1}\right) \backslash V(C)=\left\{y_{1}, y_{2}\right\}$. If $|X|=4$, then $G_{1}$ has a cycle of length at least 7 . Hence we have $|X|=2$ and let $X=\left\{w_{1}, w_{2}\right\}$. By the definition of $G_{1},\left|N_{G_{1}}\left(y_{1}\right) \cap X\right|=$ $\left|N_{G_{1}}\left(y_{2}\right) \cap X\right|=1$ and $N_{G_{1}}\left(y_{1}\right) \cap X \cap N_{G_{1}}\left(y_{2}\right)=\emptyset$. Without loss of generality, we may assume that $w_{1} \in N_{G_{1}}\left(y_{1}\right) \cap X$ and $w_{2} \in N_{G_{1}}\left(y_{2}\right) \cap X$, then by the definition of $G_{1}$, there is a vertex $w$ in $V(C) \backslash\left\{w_{1}, w_{2}\right\}$ such that $w \in N_{G_{1}}\left(y_{1}\right) \cap N_{G_{1}}\left(y_{2}\right)$. Since $C$ is a longest cycle of $G_{1}$, we have $2 \leqslant \operatorname{dist}_{C}\left(w_{i}, w\right) \leqslant 3$ for each $i \in\{1,2\}$. Suppose that $\operatorname{dist}_{C}\left(w_{i}, w\right)=3$ for some $i \in\{1,2\}$, say $i=1$. Without loss of generality, we may assume that $w_{1}=v_{0}, w=v_{3}$, then, since $C$ is a longest cycle of $G_{1}, w_{2} \notin\left\{v_{2}, v_{4}\right\}$ and thus $w_{2} \in\left\{v_{1}, v_{5}\right\}$. Without loss of generality, we may assume that $w_{2}=v_{5}$, then $E\left(G_{1}\right)=\left\{y_{1} v_{0}, y_{1} v_{3}, y_{2} v_{3}, y_{2} v_{5}\right\} \cup E(C)$, thus $G_{1} \backslash v_{0} v_{5}$ is even, a contradiction.

Hence $\operatorname{dist}_{C}\left(w_{i}, w\right)=2$ for all $i \in\{1,2\}$. Without loss of generality, we may assume that $w_{1}=v_{0}, w=v_{2}$, then $w_{2}=v_{4}$. Hence $E\left(G_{1}\right)=\left\{y_{1} v_{0}, y_{1} v_{2}, y_{2} v_{2}, y_{2} v_{4}\right\} \cup E(C)$, then $G_{1}$ has a spanning trail starting from any vertex of $\left\{v_{0}, v_{4}\right\}$. Note that for any vertex $v \in\left\{y_{1}, y_{2}, v_{1}, v_{3}, v_{5}\right\}$, there is an edge $e \in E\left(G_{1}\right)$ incident with $v$ such that $G_{1} \backslash e$ has exactly two vertices with degree odd. Since $G_{1} \backslash e$ is connected, $G_{1}$ has a spanning trail starting from any vertex of $\left\{y_{1}, y_{2}, v_{1}, v_{3}, v_{5}\right\}$. Therefore, the above facts imply that $G_{1}$ has a spanning trail starting from any vertex in $\left\{y_{1}, y_{2}, v_{0}, v_{1}, v_{3}, v_{4}, v_{5}\right\}$. However, $G_{1}$ has no spanning trail starting from $v_{2}$ and thus $u=v_{2}$, hence $G_{1} \cong F_{3}$, where $F_{3}$ is shown in Figure 2.

We claim that $G^{\prime} \cong G_{1} \cong F_{3}$. Otherwise, there is an edge $f \in E\left(G^{\prime}\right) \backslash E\left(G_{1}\right)$. Since $G^{\prime}$ is triangle-free and $C$ is a longest cycle of $G_{1}, f \in\left\{v_{0} v_{3}, v_{2} v_{5}, v_{1} v_{4}, y_{1} v_{4}, y_{2} v_{0}\right\}$, thus $G_{1} \cup\{f\}$ has a spanning trail starting from $v_{2}$. This implies that $G^{\prime}$ has a spanning trail starting from any vertex, a contradiction.

Case 1.3. $G_{1}-V(C) \cong K_{1}$.

Let $V\left(G_{1}\right) \backslash V(C)=\{z\}$. By the definition of $G_{1}, N_{G_{1}}(z)=X$ and $|X|=2$, and let $X=\left\{v_{i}, v_{j}\right\}$. Since $C$ is a longest cycle of $G_{1}$, we have $2 \leqslant \operatorname{dist}_{C}\left(v_{i}, v_{j}\right) \leqslant 3$. Suppose that $\operatorname{dist}_{C}\left(v_{i}, v_{j}\right)=3$. Without loss of generality, we may assume that $v_{i}=v_{0}, v_{j}=v_{3}$, then $E\left(G_{1}\right)=\left\{z v_{0}, z v_{3}\right\} \cup E(C)$, hence $G_{1}$ has a spanning trail starting from any vertex in $\left\{v_{0}, v_{3}\right\}$. Note that for any vertex $v$ in $V\left(G_{1}\right) \backslash\left\{v_{0}, v_{3}\right\}$, there is an edge $e \in E\left(G_{1}\right)$ 
incident with $v$ such that $G_{1} \backslash e$ has exactly two vertices with degree odd. Since $G_{1} \backslash e$ is connected, $G_{1}$ has a spanning trail starting from any vertex in $V\left(G_{1}\right) \backslash\left\{v_{0}, v_{3}\right\}$. The above facts imply that $G_{1}$ has a spanning trail starting from any vertex, a contradiction.

Hence we have $\operatorname{dist}_{C}\left(v_{i}, v_{j}\right)=2$. Without loss of generality, we may assume that $v_{i}=v_{0}, v_{j}=v_{2}$, then $E\left(G_{1}\right)=\left\{z v_{0}, z v_{2}\right\} \cup E(C)$, hence $G_{1}$ has a spanning trail starting from any vertex of $\left\{v_{0}, v_{2}\right\}$. Note that for any $v \in\left\{z, v_{1}, v_{3}, v_{5}\right\}$, there is an edge $e \in E\left(G_{1}\right)$ incident with $v$ such that $G_{1} \backslash e$ has exactly two vertices with degree odd. Since $G_{1} \backslash e$ is connected, $G_{1}$ has a spanning trail starting from any vertex of $\left\{z, v_{1}, v_{3}, v_{5}\right\}$. The above facts imply that $G_{1}$ has a spanning trail starting from any vertex of $\left\{z, v_{0}, v_{1}, v_{2}, v_{3}, v_{5}\right\}$. However, $G_{1}$ has no spanning trail starting from $v_{4}$ and then $u=v_{4}$, hence $G_{1} \cong F_{6}$, where $F_{6}$ is shown in Figure 2.

We claim that $G^{\prime} \cong G_{1} \cong F_{6}$. Suppose not. Then there is an edge $f \in E\left(G^{\prime}\right) \backslash E\left(G_{1}\right)$. Since $G^{\prime}$ is triangle-free and $C$ is a longest cycle of $G^{\prime}, f \in\left\{v_{0} v_{3}, v_{1} v_{4}, v_{2} v_{5}, z v_{4}\right\}$, thus $G_{1} \cup\{f\}$ has a spanning trail starting from $v_{4}$. This implies that $G^{\prime}$ has a spanning trail starting from any vertex, a contradiction.

Case 2. $|V(C)|=7$.

Then $G_{1}-V(C)$ has only one vertex $z$. By the definition of $G_{1}, N_{G_{1}}(z)=X$ and $|X|=2$, and let $X=\left\{v_{i}, v_{j}\right\}$. Since $C$ is a longest cycle of $G_{1}$, we have $2 \leqslant \operatorname{dist}_{C}\left(v_{i}, v_{j}\right) \leqslant$ 3. Suppose that $\operatorname{dist}_{C}\left(v_{i}, v_{j}\right)=2$. Without loss of generality, we may assume that $v_{i}=v_{0}, v_{j}=v_{2}$, then $E\left(G_{1}\right)=\left\{z v_{0}, z v_{2}\right\} \cup E(C)$, hence $G_{1}$ has a spanning trail starting from any vertex of $\left\{v_{0}, v_{2}\right\}$. Note that for any vertex $v \in\left\{v_{1}, v_{3}, v_{6}, z\right\}$, there is an edge $e \in E\left(G_{1}\right)$ incident with $v$ such that $G_{1} \backslash e$ has exactly two vertices with degree odd. Since $G_{1} \backslash e$ is connected, $G_{1}$ has a spanning trail starting from any vertex of $\left\{v_{1}, v_{3}, v_{6}, z\right\}$, hence $G_{1}$ has a spanning trail starting from any vertex of $\left\{v_{0}, v_{1}, v_{2}, v_{3}, v_{6}, z\right\}$. However, $G_{1}$ has no spanning trail starting from any vertex of $\left\{v_{4}, v_{5}\right\}$ and then $u \in\left\{v_{4}, v_{5}\right\}$, hence $G_{1} \cong F_{4}$ where $F_{4}$ is shown in Figure 2 . We claim that $G^{\prime} \cong G_{1} \cong F_{4}$. Suppose, to the contrary, and let $f$ be an edge in $E\left(G^{\prime}\right) \backslash E\left(G_{1}\right)$. Since $G^{\prime}$ is triangle-free, $f \in\left\{v_{0} v_{3}, v_{0} v_{4}, v_{1} v_{4}, v_{1} v_{5}, z v_{4}, z v_{5}, v_{2} v_{5}, v_{2} v_{6}, v_{3} v_{6}\right\}$, then $G_{1} \cup\{f\}$ has a spanning trail starting from $v_{4}$. This implies that $G^{\prime}$ has a spanning trail starting from any vertex, a contradiction.

Hence we have $\operatorname{dist}_{C}\left(v_{i}, v_{j}\right)=3$. Without loss of generality, we may assume that $v_{i}=v_{0}, v_{j}=v_{3}$, then $E\left(G_{1}\right)=\left\{z v_{0}, z v_{3}\right\} \cup E(C)$. Hence $G_{1}$ has a spanning trail starting from any vertex of $\left\{v_{0}, v_{3}\right\}$. Note that for any vertex $v \in\left\{z, v_{1}, v_{2}, v_{4}, v_{6}\right\}$, there is an edge $e \in E\left(G_{1}\right)$ incident with $v$ such that $G_{1} \backslash e$ has exactly two vertices with degree odd. Since $G_{1} \backslash e$ is connected, $G_{1}$ has a spanning trail starting from any vertex of $\left\{z, v_{1}, v_{2}, v_{4}, v_{6}\right\}$. Hence $G_{1}$ has a spanning trail starting from any vertex of $\left\{z, v_{0}, v_{1}, v_{2}, v_{3}, v_{4}, v_{6}\right\}$. However, $G_{1}$ has no spanning trail starting from $v_{5}$ and then $u=v_{5}$, hence $G_{1} \cong F_{5}$, where $F_{5}$ is shown in Figure 2. We claim that $G^{\prime} \cong G_{1} \cong F_{5}$. Suppose not, and let $f$ be an edge in $E\left(G^{\prime}\right) \backslash E\left(G_{1}\right)$. Since $G$ is triangle-free, $f \in\left\{v_{0} v_{3}, v_{0} v_{4}, v_{1} v_{5}, z v_{5}, v_{2} v_{5}, v_{3} v_{6}\right\}$, thus $G_{1} \cup\{f\}$ has a spanning trail starting from $v_{5}$. This implies that $G^{\prime}$ has a spanning trail starting from any vertex, a contradiction.

Summarizing all possible cases, we obtain that $G^{\prime} \in\left\{F_{1}, F_{2}, F_{3}, F_{4}, F_{5}, F_{6}\right\}$, where $F_{i}$ is shown in Figure 2. We claim that $G \cong G^{\prime}$. Suppose not. Then there is a vertex 
$v$ in $G^{\prime}$ which is the contraction image of some nontrivial collapsible graph $H$ of $G$, $|V(G)| \geqslant V\left(G^{\prime}\right)+|V(H)|-1 \geqslant 7+3-1=9$, because the nontrivial subgraph with smallest number of vertices is the triangle, a contradiction. This proves Theorem 6 .

\section{The proof of Theorem 5}

The proof of Theorem 5 can be separated the following two results.

Theorem 25. Let $G$ be a 2-edge-connected graph and $S$ a subset of $V(G)$ such that $E(G-S)=\emptyset$ and $|S| \leqslant 4$. Then $G$ has an $S$-trail starting from any vertex of $S$.

Theorem 26. Let $G$ be a 2-edge-connected graph and $S$ a subset of $V(G)$ such that $E(G-S)=\emptyset$ and $|S| \leqslant 6$. Then $G$ has an $S$-trail or $G \in\left\{G_{2}, G_{3}\right\}$, where $G_{2}, G_{3}$ are shown in Figure 1.

Proof of Theorem 25. Suppose, by contradiction, that $G$ is a counterexample to the theorem such that the number of blocks of $G$ is minimized. We now distinguish the following two cases to obtain our desired contradiction.

Case 1. $\kappa(G) \geqslant 2$.

Let $C=v_{1} v_{2} \cdots v_{c(G)} v_{1}$ be a longest cycle of $G$. Then $G-V(C)$ contains at least one vertex of $S$, otherwise $C$ is a Hamilton cycle. Therefore, since $|S| \leqslant 4$, we have $|V(C) \cap S| \leqslant 3$. Since $C$ is a subgraph of $G$ and $E(G-S)=\emptyset$, we have $E(C-S)=\emptyset$. Note that $G$ has no spanning trail starting from some vertex of $S$. By Theorem 4(1) we have $c(G) \geqslant 6$. If $c(G) \geqslant 7$, then $|V(C) \cap S| \geqslant 4$ since $E(C-S)=\emptyset$, contradicting $|V(C) \cap S| \leqslant 3$. Hence we have $c(G)=6$, then $|V(C) \cap S| \geqslant 3$ since $E(C-S)=\emptyset$, thus $|V(C) \cap S|=3$. We therefore assume that $V(C) \cap S=\left\{v_{1}, v_{3}, v_{5}\right\}$ and $V(C) \backslash S=$ $\left\{v_{2}, v_{4}, v_{6}\right\}$. Since $|S| \leqslant 4$ and $G-V(C)$ contains at least one vertex of $S, G-V(C)$ contains exactly one vertex of $S$, say $b$. Let $D$ be a component of $G-V(C)$ such that $b \in V(D)$. Then $S \subset V(C) \cup V(D)$. Let $G^{\prime} \cong\langle V(C) \cup V(G / D)\rangle_{G}$. Clearly, $G^{\prime}$ has exactly seven vertices. Since $G$ has no spanning trail starting from some vertex of $S$ and by the the definition of $G^{\prime}, G^{\prime}$ also has no spanning trail starting from some vertex of $S$. By Theorem 6, $G^{\prime}$ is isomorphic to $F_{6}$ shown in Figure 2. Let $U_{1}$ be the set of vertices of degree two in $F_{6}$ and $U_{2}$ the set of vertices of degree three in $F_{6}$. Then $\left\{v_{2}, v_{4}, v_{6}\right\} \subset U_{1}$ and $U_{2} \subset\left\{v_{1}, v_{3}, v_{5}\right\}$, one can easily check that $F_{6}$ has an $S$-trail starting from any vertex of $S$, a contradiction.

Case 2. $\kappa(G)=1$.

Since $E(G-S)=\emptyset$, each cycle of $G$ contains at least two vertices of $S$.

Claim 27. $|V(F) \cap S| \geqslant 3$ for any end block $F$ of $G$.

Proof. Suppose, by contradiction, that there is an end block $F$ of $G$ such that $|V(F) \cap S| \leqslant$ 2. Let $C$ be a cycle in $F$ that contains the only cut vertex of $G$ in $F$. Since $E(G-S)=\emptyset$, each cycle of $G$ contains at least two vertices of $S$, then $C$ contains all vertices in $V(F) \cap S$ since $|V(F) \cap S| \leqslant 2$. 
Consider the graph $G / F$ such that the contraction image $v$ of $F$ is in $S$. Then $|V(G / F) \cap S| \leqslant 4-2+1=3$. Note that $G / F$ has blocks less than $G$ and $G / F$ is also 2-edge-connected, by the choice of $G, G / F$ has an $S \cap V(G / F)$-trail $T$ starting from any vertex of $S$, then $T \cup C$ is an $S$-trail of $G$ since $C$ contains all vertices in $V(F) \cap S$, a contradiction. This proves Claim 27.

Let $F_{1}$ and $F_{2}$ be two end blocks of $G$. By Claim $27,\left|S \cap V\left(F_{i}\right)\right| \geqslant 3$ for $1 \leqslant i \leqslant 2$, then $|S| \geqslant\left|S \cap V\left(F_{1} \cup F_{2}\right)\right|=\left|S \cap V\left(F_{1}\right)\right|+\left|S \cap V\left(F_{2}\right)\right|-\left|S \cap V\left(F_{1} \cap F_{2}\right)\right| \geqslant 3+3-1=5$, a contradiction. This proves Theorem 25 .

We will apply Theorem 25 and the following result to prove Theorem 26 .

Theorem 28. (Niu. et. al [19]) Let $G$ be a 2-edge-connected graph of order at most ten. Then $G$ has a spanning trail or $G \in\left\{G_{2}, G_{3}\right\}$, where $G_{2}, G_{3}$ are shown in Figure 1 .

Proof of Theorem 26. Suppose, by contradiction, that $G$ is a counter-example to the theorem such that the number of blocks of $G$ is minimized. Then $G$ has no spanning trail. Let $S$ be a subset of $V(G)$ such that $E(G-S)=\emptyset$ and $|S| \leqslant 6$. We now consider two cases to obtain our desired contradiction.

Case 1. $\kappa(G) \geqslant 2$.

Let $C=v_{1} v_{2} \cdots v_{c(G)} v_{1}$ be a longest cycle of $G$. Then $G-V(C)$ contains at least two vertices of $S$, otherwise $G$ obviously has a spanning trail. Then, by $|S| \leqslant 6$, we have $|V(C) \cap S| \leqslant 4$. Since $C$ is a subgraph of $G$ and $E(G-S)=\emptyset$, we have $E(C-S)=\emptyset$. If $c(G) \leqslant 7$, then by Theorem $4(2), G$ has a spanning trail, a contradiction. If $c(G) \geqslant 9$, then $|V(C) \cap S| \geqslant 5$ since $E(C-S)=\emptyset$, contradicting $|V(C) \cap S| \leqslant 4$. Hence we have $c(G)=8$, then $|V(C) \cap S|=4$. We therefore assume that $V(C) \cap S=\left\{v_{1}, v_{3}, v_{5}, v_{7}\right\}$ and $V(C) \backslash S=\left\{v_{2}, v_{4}, v_{6}, v_{8}\right\}$. Therefore, since $G-V(C)$ contains at least two vertices of $S$ and $|S| \leqslant 6, G-V(C)$ contains exactly two vertices of $S$, say $b_{1}, b_{2}$. Then $b_{1}$ and $b_{2}$ cannot lie in a same component of $G-V(C)$; otherwise, one can easily find an $S$-trail of $G$. Let $D_{1}$ and $D_{2}$ be two components of $G-V(C)$ such that $b_{i} \in V\left(D_{i}\right)$. Then $S \subset V(C) \cup V\left(D_{1}\right) \cup V\left(D_{2}\right)$. Let $G^{\prime}=\left\langle V(C) \cup V\left(G / D_{1}\right) \cup V\left(G / D_{2}\right)\right\rangle_{G}$. Clearly, $G^{\prime}$ has exactly ten vertices. Since $G$ has no $S$-trail and by the definition of $G^{\prime}, G^{\prime}$ has no $S$-trail, implying that $G^{\prime}$ has no spanning trail. Note that $G^{\prime}$ is also 2-connected. By Theorem 28, $G^{\prime} \in\left\{G_{2}, G_{3}\right\}$, where $G_{2}, G_{3}$ are shown in Figure 1 .

Let $U_{1}$ be the set of vertices of degree two in $G^{\prime}$ and $U_{2}$ the set of vertices of degree three in $G^{\prime}$. Then $\left|U_{1}\right|=6$ and $\left|U_{2}\right|=4$. Note that $S=\left\{v_{1}, v_{3}, v_{5}, v_{7}, b_{1}, b_{2}\right\}$. Then $U_{1}=S$, otherwise, $\left|U_{2} \cap S\right| \neq \emptyset$, one can easily find an $S$-trail of $G^{\prime}$. We furthermore have $V\left(D_{i}\right)=\left\{b_{i}\right\}$ for $1 \leqslant i \leqslant 2$, otherwise contradicting $E(G-S)=\emptyset$. This implies that $G^{\prime}$ is a subgraph of $G$.

Claim 29. $V(G)=V\left(G^{\prime}\right)$.

Proof. Suppose to the contrary that there is a vertex $w \in V(G) \backslash V\left(G^{\prime}\right)$. Then there exist two edges between $w$ and $C$ in $G$ since $G$ is 2-connected. Therefore, since $G^{\prime}$ is a subgraph of $G$ and $U_{1}=S$, one can easily find an $S$-trail of $G$, a contradiction. This proves Claim 29. 
Since $G^{\prime} \subseteq G$ and by Claim $29, G^{\prime}$ is a spanning subgraph of $G$. Since $G^{\prime} \in\left\{G_{2}, G_{3}\right\}$, it is easy to check that adding any edge to $G^{\prime}$ can make it have an $S$-trail, this implies that $G \cong G^{\prime}$, contradicting the hypothesis.

Case 2. $\kappa(G)=1$.

Then $G$ has at least two blocks.

Claim 30. $|V(F) \cap S| \geqslant 3$ for any end block $F$ of $G$.

Proof. Suppose, by contradiction, that there is an end block $F$ of $G$ such that $|V(F) \cap S| \leqslant$ 2. Let $C$ be a cycle in $F$ that contains the only cut vertex of $G$ in $F$. Since $E(G-S)=\emptyset$, each cycle of $G$ contains at least two vertices of $S$, then $C$ contains all vertices in $V(F) \cap S$ since $|V(F) \cap S| \leqslant 2$.

Consider the graph $G / F$ such that the contraction image $v$ of $F$ is in $S$. Then $|V(G / F) \cap S| \leqslant 6-2+1=5$. Note that $G / F$ has blocks less than $G, G / F$ has an $S \cap V(G / F)$-trail $T$; otherwise, by the choice of $G$, the connectivity of $G / F$ is at least two, by Case 1 we have $G / F \in\left\{G_{2}, G_{3}\right\}$, where $G_{2}, G_{3}$ are shown in Figure 1 . Let $U$ be the set of vertices of degree two in $G / F$. Then $|U|=6$. Since $G / F$ has no $S \cap V(G / F)$-trail, this implies that $U \subseteq(S \cap V(G / F))$, then $|V(G / F) \cap S| \geqslant 6$, contradicting $|V(G / F) \cap S| \leqslant 5$. Therefore, $v \in \bar{V}(T), T \cup C$ is an $S$-trail of $G$ since $C$ contains all vertices in $V(F) \cap S$, a contradiction. This proves Claim 30 .

Claim 31. G has exactly two end blocks.

Proof. Suppose, to the contrary, that $G$ has three end blocks $F_{1}, F_{2}$ and $F_{3}$. By Claim 30, $\left|S \cap V\left(F_{1} \cup F_{2} \cup F_{3}\right)\right|=\left|S \cap V\left(F_{1}\right)\right|+\left|S \cap V\left(F_{2}\right)\right|+\left|S \cap V\left(F_{3}\right)\right|-\left|S \cap V\left(F_{1} \cap F_{2}\right)\right|-\mid S \cap$ $V\left(F_{1} \cap F_{3}\right)|-| S \cap V\left(F_{2} \cap F_{3}\right)|+| S \cap V\left(F_{1} \cap F_{2} \cap F_{3}\right) \mid \geqslant 3+3+3-1-1-1+1=7$, a contradiction. This proves Claim 31.

By Claim 31, let $F_{1}$ and $F_{2}$ be all end blocks of $G$ such that $v_{i}$ is the cut vertex of $G$ in $F_{i}$. By Claim $30,\left|V\left(F_{i}\right) \cap S\right| \geqslant 3$ for $1 \leqslant i \leqslant 2$. Since $|S| \leqslant 6$, we have $3 \leqslant\left|V\left(F_{i}\right) \cap S\right| \leqslant 4$ for $1 \leqslant i \leqslant 2$. Indeed, $F_{1} \cup F_{2}$ contains all vertices of $S$. Let $P$ be a path in $G$ connecting $v_{1}$ and $v_{2}$ (possibly $P$ is a vertex). For $i \in\{1,2\}, F_{i}$ satisfies the conditions of Theorem 25, $F_{i}$ has an $S \cap V\left(F_{i}\right)$-trail starting from any vertex of $S$. Note that $v_{i} \in S$ or $v_{i}$ is adjacent to some vertex in $S$ for each $i \in\{1,2\}$. This implies that $F_{i}$ has an $S \cap V\left(F_{i}\right)$-trail $T_{i}$ starting from $v_{i}$ for $i=1,2$, hence $T_{1} \cup P \cup T_{2}$ is an $S$-trail of $G$, a contradiction. This proves Theorem 26.

\section{Proofs of Theorem 7 and Corollary 8}

The core of a graph $G$, denoted by $G_{0}$, is obtained by recursively deleting all pendant vertices in $G$. The clique covering number of a graph $G$, denoted by $\theta(G)$, is the minimum number of cliques necessarily that cover $V(G)$. Color the vertices of a graph $G$ black and white, we use $B(G)$ and $W(G)$ to denote the set of black vertices and whites vertices in $G$, respectively. A star decomposition of a graph $G$ is a family $\mathcal{F}$ of edge-disjoint stars $F$ of $G$ such that $\bigcup_{F \in \mathcal{F}} E(F)=E(G)$. 
Theorem 32. (Li, et al. [17]) Let $G$ be a graph with $|E(G)| \geqslant 3$. Then the line graph $L(G)$ is traceable if and only if $G$ has a dominating connected subgraph, i.e., dominating trail.

Lemma 33. (Chvátal-Erdös, [7]) Every connected graph $G$ of order at least three with $\alpha(G) \leqslant \kappa(G)+1$ is traceable.

Lemma 34. (Kasier, et al. [13]) Every 5-connected claw-free graph with minimum degree at least 6 is hamiltonian.

Lemma 35. (Favaron, et al. [9]) Let $k \geqslant 2$ be an integer and let $G$ be a claw-free graph of order $n \geqslant 2 k^{2}-3 k$ and minimum degree $\delta(G)>\frac{n}{k}+k-2$. Then $\theta(\operatorname{cl}(\mathrm{G})) \leqslant \mathrm{k}-1$.

Lemma 36. (Fronček, et al. [10]) Let $k \geqslant 2$ be an integer, let $G$ be a claw-free graph of order $n$ and let $\kappa=\kappa(\mathrm{cl}(\mathrm{G}))$. Suppose that $G$ is such that $n \geqslant 3 k^{2}-k-\kappa-2$ and $\delta(G)>\frac{n+k^{2}-4 k+2+\kappa}{k}$. Then $\theta(\operatorname{cl}(\mathrm{G})) \leqslant \mathrm{k}-1$, or $\left.\alpha(\mathrm{cl}(\mathrm{G}))\right) \leqslant \kappa$.

Theorem 37. Let $G$ be a 2-connected closed claw-free graph with $\theta(G) \leqslant 6$. Then $G$ is traceable or $G=L(H)$ where $H \in \mathcal{C}_{1} \cup \mathcal{C}_{2}$.

Proof. Let $G$ be a closed claw-free graphs with clique covering number $\theta$. Let $\mathcal{P}_{G}=$ $\left\{B_{1}, \cdots, B_{\theta}\right\}$ be a clique covering of $G$ such that each $B_{i}$ is maximal. Then $H=L^{-1}(G)$ has a star decomposition $T_{1}, \cdots, T_{\theta}$ such that $L\left(T_{i}\right) \cong B_{i}$. Color black on those centers $c_{B_{i}}$ of $T_{i}$ and white on remaining vertices in $V(H) \backslash\left\{c_{B_{1}}, \cdots, c_{B_{t}}\right\}$. Then $B(H)=$ $\left\{c_{B_{1}}, \cdots, c_{B_{\theta}}\right\}$. Note that $B(H)$ is a vertex covering of $H$ (that is, every edge of $H$ has at least one vertex in $B(H)), E(H-B(H))=\emptyset$.

Suppose that $G$ is non-traceable. It suffices to show that $H \in \mathcal{C}_{1} \cup \mathcal{C}_{2}$. By the definition of the core $H_{0}$, the black vertices of $H$ are alway black in $H_{0}$, i.e., $B(H)=B\left(H_{0}\right)$. Since $G$ is non-traceable and by Theorem $32, H_{0}$ has no $B\left(H_{0}\right)$-trail. Since $\theta(G) \leqslant 6$, we have $|B(H)| \leqslant 6$ and then $\left|B\left(H_{0}\right)\right| \leqslant 6$. Since $H_{0}$ is a subgraph of $H$ and $E(H-B(H))=\emptyset$, we have $E\left(H_{0}-B\left(H_{0}\right)\right)=\emptyset$. Since $H_{0}$ has no $B\left(H_{0}\right)$-trail and by Theorem $5, H_{0} \in\left\{G_{2}, G_{3}\right\}$, where $G_{2}, G_{3}$ are shown in Figure 1. Let $U_{1}$ be the set of vertices of degree two in $H_{0}$ and $U_{2}$ the set of vertices of degree three in $H_{0}$. Then $\left|U_{1}\right|=6$ and $\left|U_{2}\right|=4$.

If $U_{2} \cap B\left(H_{0}\right) \neq \emptyset$, then, since $\left|B\left(H_{0}\right)\right| \leqslant 6$ and $\left|U_{1}\right|=6$, we have $U_{1} \backslash B\left(H_{0}\right) \neq \emptyset$, say $u \in U_{1} \backslash B\left(H_{0}\right)$, it is easy to see that $H_{0}-u$ has a spanning trail, then $H_{0}$ has a $B\left(H_{0}\right)$ trail, a contradiction. Hence $U_{2} \cap B\left(H_{0}\right)=\emptyset$ and then $B\left(H_{0}\right) \subseteq U_{1}$, hence $B\left(H_{0}\right)=U_{1}$; otherwise, one can easily check that $H_{0}$ has a $B\left(H_{0}\right)$-trail, a contradiction. Therefore, every vertex in $U_{1}$ has at least one pendant vertex in $H$, implying that $H \in \mathcal{C}_{1} \cup \mathcal{C}_{2}$. This proves Theorem 37.

The following result is a consequence of Theorem 37 .

Corollary 38. Let $G$ be a 2-connected closed claw-free graph with $\theta(G) \leqslant 5$. Then $G$ is traceable. 
Proof of Theorem \%. Suppose that $G$ is non-traceable. Then by Theorem $10, \mathrm{cl}(\mathrm{G})$ is also non-traceable. By Lemma 33, $\alpha(\operatorname{cl}(\mathrm{G}))>\kappa(\operatorname{cl}(\mathrm{G}))+1$. Thus, Lemma 34 implies $1 \leqslant \kappa(\mathrm{cl}(\mathrm{G})) \leqslant 5$. The assumptions of theorem satisfy the conditions of Lemma 36 (for $k=7)$, we then obtain $\theta(\operatorname{cl}(\mathrm{G})) \leqslant 6$, by Theorem $37, \operatorname{cl}(\mathrm{G})=\mathrm{L}(\mathrm{H})$ where $H \in \mathcal{C}_{1} \cup \mathcal{C}_{2}$. This proves Theorem 7 .

Proof of Corollary 8. Let $G$ satisfy the assumptions of Corollary 8. Then clearly so is $\mathrm{cl}(\mathrm{G})$. Thus, suppose that $G$ is closed. From Lemma 35 (for $k=6$ ) we then obtain $\theta(G) \leqslant 5$. By Corollary 38, $G$ is traceable. This proves Corollary 8 .

\section{Concluding remarks}

In this paper, we prove our main result (Theorem 4), we also use it to obtain Theorems 5 and 6. By using Theorem 5 and more detailed discussion as Theorem 7, Tian et al. [22] gave a sharp slight weaker minimum degree sum of a pair of adjacent vertices for those 2connected claw-free graphs with minimun degree at least three to be traceable, and Tian et al. [23] gave also a sharp minimun degree sum of $t$ independent vertex set, however, these 2-connected claw-free traceable graphs deduced by these conditions have some exceptional graphs obtained from $G_{2}, G_{3}$ shown in Figure 1.

We believe that Theorem 4 would have more applications. As Theorem 2 was used in the forbidden subgraph condition for a 3 -connected claw-free $H$-free graph to be hamiltonian, it will be also used in the similar forbidden subgraph condition for a 2-connected claw-free graph to be traceable in our futher work [24].

\section{Acknowledgements}

This work is supported by Natural Science Foundation of China (Nos. 11871099, 11671037) and Natural Science Foundation of Qinghai Province (No. 2018-ZJ-717)

\section{References}

[1] J. A. Bondy and U. S. R. Murty. Graph Theory, Graduate in Mathematics. Springer, 2008.

[2] S. Brandt, O. Favaron and Z. Ryjáček. Closure and stable hamiltonian properties in claw-free graphs. J. Graph Theory, 34(1):30-41, 2000.

[3] P. Catlin. Spanning trails. J. Graph Theory, 11(2):161-167, 1987.

[4] P. Catlin. A reduction mathod to find spanning eulerian subgraphs. J. Graph Theory, 12(1):29-44, 1988.

[5] G. Chen, M. Ellingham, A. Saito and S. Shan. Spanning trails with maximum degree at most 4 in $2 K_{2}$-free graphs. Graphs Combin., 33(5):1095-1101, 2017. 
[6] Z.-H. Chen, H.-J. Lai, X. Li, D. Li and J. Mao. Eulerian Sugraphs in 3-edge-connected graphs and Hamiltonian Line Graphs. J. Graph Theory, 42(4):308-319, 2003.

[7] V. Chvátal and P. Erdős. A note on Hamilton circuits. Discrete Math., 2:111-113, 1972.

[8] M. Ellingham, X. Zha and Y. Zhang. Spanning 2-trails from degree sum conditions. J. Graph Theory, 45(4):298-319, 2004.

[9] O. Favaron, E. Flandrin, H. Li and Z. Ryjáček. Clique covering and degree conditions for hamiltonicity in claw-free graphs. Discrte Math., 236(1-3):65-80, 2001.

[10] D. Fronček, Z. Ryjáček and Z. Skupień. On traceability and 2-factor in claw-free graphs. Discuss. Math. Graph Theory, 24(1):55-71, 2004.

[11] F. Harary and C. Nash-Williams. On eulerian and hamiltonian graphs and line graphs. Canad. Math. Bull., 8(6):701-710, 1965.

[12] F. Jaeger. A note on sub-eulerian graphs. J. Graph Theory, 3(1):91-93, 1979.

[13] T. Kaiser and P. Vrána. Hamilton cycles in 5-connected line graphs. European J. Combin., 33(5):924-947, 2012.

[14] O. Kovářík, M. Mulač and Z. Ryjáček. A note on degree conditions for hamiltonicity in 2-connected claw-free graphs. Discrete Math., 244(1-3):253-268, 2002.

[15] H.-J. Lai, X. Li, H. Poon and Y. Ou. Spanning trails connecting given edges. Graphs Combin., 21(1):77-88, 2005.

[16] H.-J. Lai, L. Xiong, H. Yan and J. Yan. Every 3-connected claw-free $Z_{8}$-free graph is Hamiltonian. J. Graph Theory, 64(1):1-11, 2010.

[17] D. Li, H.-J. Lai and M. Zhan. Eulerian subgraphs and hamilton-connected line graphs. Discrete Appl. Math., 145(3):422-428, 2005.

[18] W. Luo, Z.-H. Chen and W.-G. Chen. Spanning trails containing given edges. Discrete Math., 306(1):87-98, 2006.

[19] Z. Niu and L. Xiong. Smallest 2-edge-connected graphs without a spanning trail. Utilitas Mathematica, 88:381-397, 2012.

[20] Z. Ryjáček. On a closure concept in claw-free graphs. J. Combin. Theory Ser. B, 70(2):217-224, 1997.

[21] Z. Ryjáček. P. Vrána and S. Wang. Closure for $\left\{K_{1,4}, K_{1,4}+e\right\}$-free graphs. $J$. Combin. Theory Ser. B, 134 (2019):239-263.

[22] T. Tian, L. Xiong, Z.-H. Chen and S. Wang. Degree sums of adjacent vertices for traceability of claw-free graphs, submitted.

[23] T. Tian, H. Broersma and L. Xiong. A note on sufficient degree conditions for traceability of claw-free graphs, submitted.

[24] S. Wang and L. Xiong. Forbidden pairs for traceability of 2-connected graphs, submitted.

[25] L. Xiong and M. Zong. Traceability of line graphs. Discrete Math., 309(12):37793785, 2009. 